\title{
CHANGES IN RENAL FUNCTION AND PERSISTENCE OF THE MURMUR AFTER LIGATURE OF A, PATENT DUCTUS ARTERIOSUS
}

\author{
BY \\ GEOFFREY BOURNE
}

Received July 24, 1941

When first seen the patient here referred to was suffering from infection of a patent ductus arteriosus by Streptococcus viridans. Ligature of the ductus and treatment by sulphapyridine effected a cure. Full clinical and operative details are described, together with a second case of patent ductus arteriosus infected by $B$. influenze and cured by ligation and chemotherapy, in a paper by Bourne, Keele, and Tubbs (1941).

The reason for the present publication is the existence of the following two points of interest, additional to the fact of cure, points which seem to deserve separate and full description :

(I) As a result of obliteration of the ductus there resulted a temporary but striking and prolonged impairment of renal function, associated with a marked increase of the diastolic blood pressure ;

(II) In spite of obliteration of the ductus the typical murmur persisted for some months.

The patient, a girl of 19, had been in good health until four months previous to her admission to St. Bartholomew's Hospital. She had suffered during this time from headache, lassitude, and undue sweating at night. Fever was present throughout this period. No peripheral or pulmonary embolic symptoms were reported or found. The heart was enlarged, especially to the left. The typical systolic thrill and murmur of patent ductus arteriosus were present. The blood pressure was $132 / 40$. The pulmonary conus was seen radiographically to be enlarged. Blood culture proved on several occasions the presence of Streptococcus viridans. The growth became progressively less profuse with sulphapyridine therapy, and before operation the blood was sterile.

Since the ligation of the ductus, and for a period of nine months, she has remained perfectly well.

The following sentences are quoted from Mr. Tubbs' account of the surgical procedure, with slight abbreviation and modification. The ductus arteriosus was approached through the left pleural cavity, which was opened through the second left intercostal space, the skin incision extending from the mid-line in front to the anterior axillary line. The second and third costal cartilages were 
divided close to the sternum, and excellent exposure was obtained by separating the ribs with a " rib-spreader". The internal mammary vessels were divided between ligatures so that full use of the medial end of the incision might be obtained. The mediastinal pleura was incised parallel and posterior to the phrenic nerve, which was then retracted anteriorly. No difficulty was experienced in identifying the patent ductus. The cellular tissue under the arch of the aorta was gently swept from the anterior surface of the ductus with a pledget of gauze held in a long hæmostatic clamp. Keeping immediately against the wall of the ductus, the adjacent tissues were readily separated by blunt dissection, except from the postero-medial surface, where a plane of cleavage was found by tactile means and without visualization. This was done by insinuating the left index finger into the cleft between the aortic arch and the bifurcation of the pulmonary artery, anterior to the ductus. None but a trivial amount of hæmorrhage was encountered. The ductus was three-quarters of an inch in length and rather more then half an inch in diameter. It was obliterated by two doubled No. 5 tubular silk ligatures.

\section{Renal Functions and Blood Pressure Changes}

Previous to operation the average blood pressure reading was $130 / 40$, and the urine, which contained no albumin or cells or casts, was sterile on culture. After the operation, and on the same day, the systolic blood pressure fell to 112 , but in five hours rose to 130 , the corresponding diastolic figures being 84 and 94 . On the following day the figures were at first 136/116, and later $116 / 100$. On the second and third days there was little change, but on the fourth day after operation the figures were 154/122 and 150/120. From this point the pressures slowly fell, reaction of $140 / 105$ on the eleventh day. The systolic pressure then became reduced during the next week to between 130 and 140, where it remained, but the diastolic continued in the neighbourhood of 94 , and not until six weeks after operation did it fall to the final permanent figure of 74 to 78 .

During the whole of this post-operative phase no abnormal cells, no casts, and no albumin were found in the urine, but there was a gross diminution in renal efficiency. Eight days following operation the urea content of the blood was $62 \mathrm{mg}$. per 100 c.c., and fifteen days afterwards this had increased to $72 \mathrm{mg}$. per 100 c.c. On this day the standard urea clearance was 21.9 c.c., or 29.2 per cent of the average normal for the first hour, and $15 \cdot 2$ c.c., or 28 per cent, for the second hour. No urinary abnormality was found even at this stage. The urea clearance figures then slowly increased, so that on the twenty-third day after operation the figures were 53.5 per cent of the average normal for the first hour and 40.8 per cent for the second.

Two months later the respective figures were 66.6 per cent and 60.3 per cent. This renal failure was closely parallel in degree with the increase and subsequent return to normal of the diastolic blood pressure, and it had the characteristics of pure glomerular failure. 


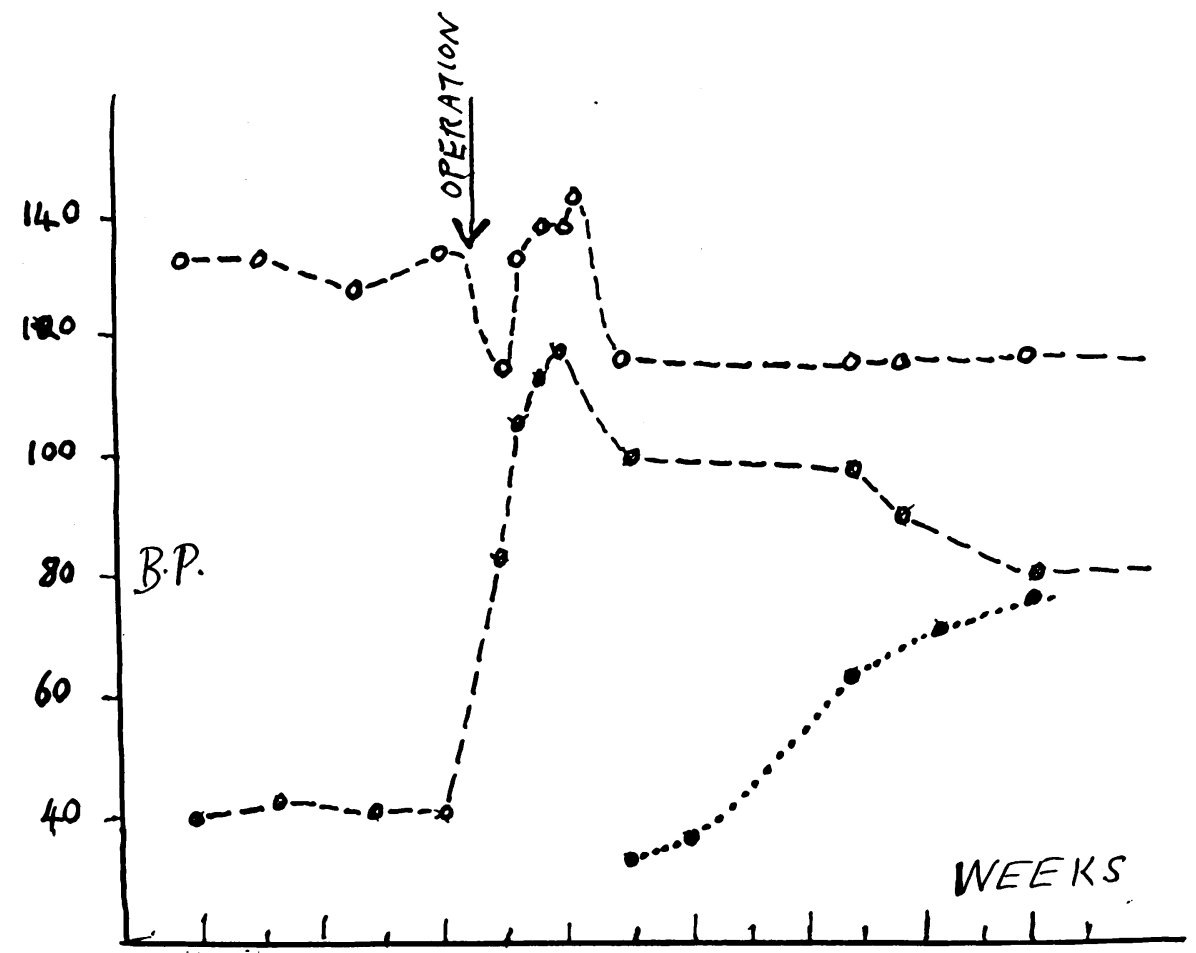

FIG. 1.-Changes in the blood pressure and renal function after ligation of a patent ductus arteriosus.

Divided lines : blood pressure, systolic and diastolic.

Dotted line : renal function, urea clearance, percentage of average normal.

The following hypothesis is offered to explain why the blood pressure and renal changes may have occurred.

Before operation the state of the cardiovascular system was as follows : The left ventricle was hypertrophied. The output per beat from this chamber was greater than normal. The total blood volume was larger than normal (Gross, 1939). The pulse curve was that of aortic incompetence, in which the peripheral arterioles were in a condition of more or less permanent partial relaxation. This cardiovascular state was normal for the patient, having been present for the whole of her nineteen years of life.

After operation the volume of the blood would have adjusted itself within a few days, and this can hardly be considered as a factor of significance. The left ventricular hypertrophy would remain for a number of weeks but might then disappear ; for when hypertrophy of the heart is produced in rabbits by experimental arterio-venous aneurysm it has been observed to disappear when the leak is closed again (personal communication, Dr. A. N. Drury). This has also been observed clinically in cases of arterio-venous aneurysm (Hitzig and Master, 1935). But during these weeks the ventricular output per beat might be expected to be increased above normal. This alone, however, could hardly be responsible for the renal and blood pressure changes.

It would seem that attention should therefore be directed to the state of 
the peripheral arterioles. For the whole of the patient's life the nervous tone of the peripheral vessels had been adjusted for the arterial leak from the patent ductus ; and also stabilized as regards their neuro-muscular mechanism to react to the type of pulse curve found characteristically in aortic regurgitation. The vessel would thus have been in that state of permanent partial dilatation associated with a lowered diastolic blood pressure.

It is suggested that the effect of the increased output per beat upon this hitherto constantly relaxed vascular bed could produce a reflex over-reaction. This abnormally hypertonic state of the arterioles might be expected to continue until the vasomotor nervous system had readjusted itself to the new conditions, or until the hypertrophy of the left ventricle had become sufficiently resolved. The period of readjustment might persist for some weeks.

If this vascular spasm involved the glomerular as well as the systemic arterioles, a single explanation is available for the abnormally high diastolic pressure following operation, for the coexisting diminution in renal function, and for the fact that the urine at no time showed any cells, casts, or albumin.

It may be objected that the renal changes had no association with the tying of the ductus and the subsequent blood pressure variations but were the direct result of the infection; and that when the infection was cured they naturally disappeared. In answer to this are the facts that although the blood urea was at one time $72 \mathrm{mg}$. per 100 c.c., at no time were red blood cells, casts, or albumin found in the urine, as is usually the case when kidney damage during endocarditis or septicæmia is extensive; and no embolic signs were present elsewhere. Nor would recovery in such a case have been complete in so short a period. Finally, the relation between the renal and blood pressure changes is obviously a close one.

After ligature of the ductus in Case I (Bourne et al., 1941) the diastolic pressure rose from 60 to 120, but later fell to the region of 80 .

Similar elevation of diastolic pressure is characteristic of closure of arteriovenous aneurysms, both in man and in experimental animals, as Holman (1940) has pointed out in a full review of the subject.

\section{Persistence of The Murmur}

The murmur of patent ductus arteriosus is typically one that fills systole and is carried on into diastole. During this period the pressure in the aorta is at first systolic, and then, in proportion to the size of the arterial leak, it falls to a lower diastolic level than normal. The murmur is loudest at those periods of the cardiac cycle when the flow of blood from aorta to pulmonary artery is most rapid. This provides the classical explanation that the murmur is directly caused by this flow. In the case here described the murmur before operation was classical, and the aortic leak was large, but after ligature of the ductus the murmur persisted, although the leak was stopped. The blood pressure was completely changed by the operation, the figures before operation being 132/40, and after operation 136/116 at first and 130/80 finally. Two doubled tubular silk ligatures were used, and there is no reason to doubt that 
they were effective. The persistence of the murmur was verified by four independent observers. It remained definite for three months, and six months later was far less loud, and its diastolic part is now (after nine months) nearly inaudible. Touroff (1940) noticed the same persistence of the murmur in two of his cases after ligature, but the length of its duration was unstated. In one of his cases the ductus was cut between two ligatures, thus disposing of a possible criticism suggesting that the ligation had been imperfect. It is clear that in all these cases the classical murmur was not caused in the manner usually suspected. It is therefore possible that it is never so caused ; its mode of production must therefore remain a matter for conjecture. Ligature of the patent ductus did, however, result in disappearance of the murmur in our Case 1 (Bourne et al., 1941). Moreover Gross (1939), after the ligature of four non-infected cases, noted persistence of the double murmur, but with diminished loudness, in two cases, persistence of a soft systolic murmur in one case, and disappearance of the murmur in one case. At operation a faint systolic thril could be palpated in the writer's case in the pulmonary conus after applying the first ligature, but after the second this was abolished.

\section{SUMmaRY}

A case of patent ductus arteriosus is described, in which ligature of the ductus

(1) caused a marked increase in the diastolic pressure, associated with great impairment of renal function, and

(2) did not result in disappearance of the classical murmur.

The hitherto undescribed changes in renal function and the persistence of the murmur, after ligature, are reported so that in future cases these points may be further investigated. Careful renal function studies should be done before as well as after operation in such cases.

\section{REFERENCES}

Bourne, G., Keele, K. D., and Tubbs, O. S. (1941). Lancet $(2,444)$. Gross, R. E. (1939). Ann. Surg. 110, 321.

Harrison, T. R., Dock, W., and Holman, E. (1924). Heart, 11, 337.

Hitzig, W. M., and Master, A. M. (1935). Mount Sinai Hosp., 1, 269.

Holman, E. (1940). Ann. Surg. 112, 840.

Touroff, A. S. W., and Vesell, H. (1940). J. Amer. med. Ass., 115, 1270.

Touroff, A. S. W. (1940). J. thorac. Surg. 10, 59. 